\title{
Evaluation of Acceleration and Deceleration Cardiac Processes using Phase-Rectified Signal Averaging in Healthy and Idiopathic Dilated Cardiomyopathy subjects
}

Rosana Bas ${ }^{\mathrm{a}}$, Montserrat Vallverdú ${ }^{\mathrm{a}}$, Jose F. Valencia ${ }^{\mathrm{b}}$, Andreas Voss ${ }^{\mathrm{c}}$, Antonio Bayés de Luna ${ }^{\mathrm{d}}$, Pere Caminal $^{\text {a }}$

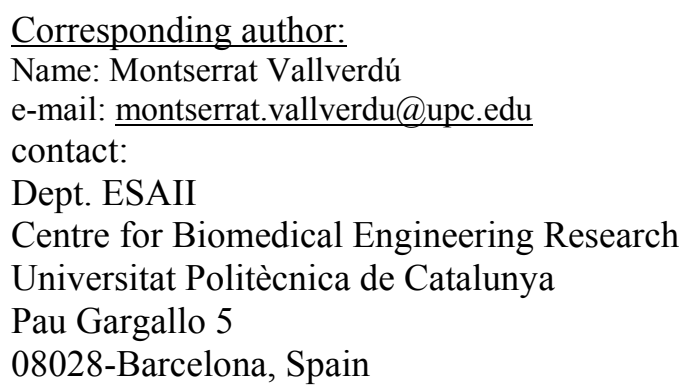

Keywords: Acceleration and deceleration capacities, autonomic nervous system, heart rate variability, sudden cardiac death

a Dept. ESAII, Centre for Biomedical Engineering Research, CIBER-BBN, BarcelonaTech, Barcelona, Spain ${ }^{b}$ Dept. Electronic Engineering, University San Buenaventura, Cali, Colombia

${ }^{\mathrm{d}}$ Catalan Institute of Cardiovascular Sciences, Barcelona, Spain 


\section{ABSTRACT}

The aim of the present study was to investigate the suitability of the Phase-Rectified Signal Averaging (PRSA) method for improved risk prediction in cardiac patients. Moreover, this technique, which separately evaluates acceleration and deceleration processes of cardiac rhythm, allows the effect of sympathetic and vagal modulations of beat-to-beat intervals to be characterized. Holter recordings of idiopathic dilated cardiomyopathy (IDC) patients were analyzed: high-risk (HR), who suffered sudden cardiac death (SCD) during the follow-up; and low-risk (LR), without any kind of cardiac-related death. Moreover, a control group of healthy subjects was analyzed. PRSA indexes were analyzed, for different time scales $T$ and wavelet scales $s$, from RR series of $24 \mathrm{~h}-\mathrm{ECG}$ recordings, awake periods and sleep periods. Also, the behavior of these indexes from simulated data was analyzed and compared with real data results. Outcomes demonstrated the PRSA capacity to significantly discriminate healthy subjects from IDC patients and HR from LR patients on a higher level than traditional temporal and spectral measures. The behavior of PRSA indexes agrees with experimental evidences related to cardiac autonomic modulations. Also, these parameters reflect more regularity of the autonomic nervous system (ANS) in HR patients.

\section{Introduction}

Heart rate changes on characteristic time-scales can be associated with regulatory actions of different branches of the autonomic nervous system (ANS). As it is known, heart rate variability (HRV) is affected by both vagal and sympathetic modulation of the sinus node. Autonomic dysfunction is closely related to cardiac mortality and susceptibility to life-threatening arrhythmic events[1]. Consequently, assessment of HRV has been proposed for risk prediction.

Due to the complex interaction between the autonomic control system and several regulatory mechanisms of heart rate, and the fact that many biological systems have an intrinsically nonlinear behavior, it is reasonable to assume that the control system regulating the heart is affected by several nonlinear variables[2]. In fact, some evidences suggest that nonlinear methods may provide supplementary information about various physiological systems involved in cardiovascular pathology[3] and may be superior predictors of cardiac dysfunction, including ventricular tachycardia and sudden cardiac death (SCD)[4,5], when compared to traditional time-domain and frequency-domain analyses. Because of this, there has been an increasing emphasis on applying nonlinear analyses to characterize the variability in cardiovascular processes, including the use of detrended fluctuation analyses, approximate entropy, sample entropy, Poincaré plots and symbolic dynamics[6,7].

Since the incidence and progression of heart failure are associated with an increasing severity of autonomic disorders, specifically a compensatory increase in activity of the sympathetic nervous system and decrease in activity of the parasympathetic or vagal nervous system[8,9], it is interesting to separately evaluate both branches of ANS. Over the past decades, power spectral analysis of HRV signals has become a commonly used tool for assessing the effect of sympathetic and 
vagal modulations of RR intervals in a non-invasive manner[10-12]. However, this analysis does not always provide reliable results, because HRV signals are often contaminated by noise and artifacts and spectral analysis does not take into account the aforementioned intrinsic nonlinear behavior of the system. Nevertheless, an approximate distinction of the effects of vagal and sympathetic factors might be made possible by the Phase-Rectified Signal Averaging (PRSA) method[13] that offers the possibility to carry out the selective analysis of acceleration-related and deceleration-related behavior of heart rate. PRSA algorithm is a nonlinear signal transformation, robust against artefacts and ectopic beats since it is able to extract periodicities and quasi-periodicities from complex signals[13,14]. This technique might provide more differentiated insights into nonlinear mechanisms involved in ANS regulation.

The aim of this study is threefold. The first is to apply the PRSA technique in order to investigate its suitability for improving risk prediction in cardiac patients from $24 \mathrm{~h}-\mathrm{ECG}$ recordings. Since idiopathic dilated cardiomyopathy (IDC) is a common and largely irreversible form of heart muscle disease[15], a group of IDC patients was analyzed and compared with a control group. The second is to characterize differences on sympathetic and vagal effects of beat-to-beat intervals by PRSA indexes derived from 24-hour Holter recordings in healthy subjects and IDC patients with high and low-risk of suffering SCD. And the third is to evaluate acceleration and deceleration patterns during awake and sleep periods. In order to delve into this issue, besides analyzing the experimental signals, the proposed methodology has been applied to simulated signals.

\section{Materials and Methods}

\subsection{Analyzed Data and Pre-Processing}

The analyzed experimental data set belongs to the IDEAL database[16]. Two groups of subjects have been selected for the present study, 62 healthy subjects (NRM) and 42 patients with IDC. Furthermore, two groups of IDC patients have been analyzed: 30 low-risk patients (LR), without any kind of cardiac-related death in the 3 years following the ECG recording, and 12 high-risk patients (HR), who suffered SCD during the follow-up after the ECG recording. The characteristics of the subjects included in the study are shown in Table I. Besides demographic characteristics, etiological aspects including the percentage of patients with ventricular tachycardia $(V T)$ and the left ventricular ejection fraction $(L V E F)$ are shown in that table. Furthermore, data related to possible comorbidities of the patients and their medication has been included. All subjects gave their written informed consent before study participation.

The RR intervals, time interval between consecutive heart beats of normal sinus rhythm, of the subjects were obtained from digital three-orthogonal-lead 24-hour Holter recordings with a sampling frequency of $200 \mathrm{~Hz}[17]$. All analyzed subjects had less than 100 ectopic beats[18]. Therefore, a possible alteration of the results due to a filter procedure can be discarded. In this way, 
RR time series were filtered by replacing artifacts or ectopic beats if they deviated more than a programmed tolerance of $15 \%$ from the mean values of the previous five beats[19,20].

Besides that, the influence of awake and sleep periods on the results has been investigated to get more detailed information about acceleration and deceleration patterns. Sleep periods correspond, approximately, to the time interval from 12:00a.m. to 6:00a.m. Meanwhile, awake periods were chosen, approximately, from 9:00a.m. to 9:00p.m.

Two types of simulated data were considered: Gaussian white noise (GWN) and autoregressive (AR) processes. GWN was utilized to simulate a fully unpredictable process; it was generated from series of pseudo-random numbers with null mean and unit standard deviation. Second-order AR processes driven by GWN were utilized to simulate partially predictable processes. Two different AR processes were obtained; one of them was shaped to have a power spectrum peak with central frequency at $0.275 \mathrm{~Hz}$ (HF band) and the other with central frequency at $0.0975 \mathrm{~Hz}$ (LF band). Both AR processes were designed with a pair of complex and conjugated poles with modulus $\rho=0.98$. These processes will be indicated as $A R H F c$ and $A R L F c$, respectively. Fifty realizations of 100000 samples were considered for each type of process.

\subsection{Analysis of the HRV}

The PRSA technique, proposed by Bauer et al.[13], was applied to characterize acceleration and deceleration processes of beat-to-beat intervals. The details of the analysis are reported in the Supplementary Data.

Increase and decrease in heart rate are detected over two time scales: time scale $T$, used to determine the window size for comparing heart rates, and wavelet scale $s$. In this study, PRSA waveforms for $T=\{1, \ldots, 150\}$ were computed and acceleration and deceleration capacities ( $A C$ and $D C$, respectively) for $s=\{1, \ldots, 150\}$ were calculated. Moreover, the length of the windows around each anchor $(2 L)$ had to be selected. Different values of $L$ were tested, $L=\{5,50,150,500\}$ samples. The analysis requires to determine the values of $T$ and $s$ that provide the best statistical diagnosis values of $A C[T, s]$ and $D C[T, s]$. The selection of the best parameters was done for each analyzed case (NRM vs.IDC and HR vs.LR from 24h-ECG recordings, awake periods and sleep periods). Moreover, the indexes $A C[1,2]$ and $D C[1,2]$, proposed by Kantelhardt et al.[14], were evaluated for all analyses.

In addition to $A C$ and $D C$ measures, the range of excursion of PRSA waveforms (acceleration excursion, $A E[T]$, and deceleration excursion, $D E[T])$ and areas under acceleration and deceleration curves $(A A[T]$ and $D A[T])$ for each selected optimal value of $T$ were calculated. These indexes are defined in Fig. 1.

The standard temporal and spectral HRV measures were also calculated according to Task Force guidelines[21]. The following HRV time-domain measures were obtained: mean of the RR intervals (meanRR) and standard deviation of the NN intervals $(S D N N)$. Furthermore, power spectra were calculated and the following normalized indexes were obtained: $H F n$, in 
high frequency band $(0.15-0.4 \mathrm{~Hz}) ; L F n$, in low frequency band $(0.04-0.15 \mathrm{~Hz})$; and $V L F n$, in a very low frequency band $(0.003-$ $0.04 \mathrm{~Hz})$. Moreover, the ratio between low frequency and high frequency power $(L F / H F)$ was calculated.

\subsection{Statistical Analysis}

The Mann-Whitney U test, a non-parametric test, was used to statistically analyze differences between the groups of subjects: NRM vs.IDC and LR vs.HR. The statistical significance level was assumed at $p$-value $<0.05$ in all the analysis. Pearson correlation coefficient $(\rho)$ was calculated between PRSA indexes $(A C$ and $D C)$ and $S D N N$, since this last diagnostic parameter is clinically relevant.

Owing to the relatively small number of patients with SCD to establish differentiated training and test groups, the results obtained in the present study should be considered exploratory. In this way, for the classification of the subjects in the groups, a quadratic discriminant function was performed on each index and the leave-one-out cross-validation technique was applied for validation. The function was estimated on all-but-one- observation of the data, and then the estimated function was used to forecast the remaining observation. This procedure was repeated for each proposed index and the number of subjects correctly classified was assessed by means of the following diagnostic test indexes: sensitivity (sen) and specificity (spe). In addition, Receiver Operator Characteristic curves $(R O C)$ were constructed and the Area Under the ROC Curves $(A U C)$ calculated with a 95\% confidence interval (CI), whereby significance was inferred from a lower bound above $A U C=0.5$.

\section{Results}

\subsection{Analysis of Parameters: $[T, s]$ and $L$}

Results of the window length test showed that both $A C$ and $D C$ indexes have very little dependence on $L$ value, lower than $0.01 \%$. From this observation, the parameter $L=150$ samples was chosen; that is, surroundings of $2 L=300$, which corresponds to intervals of approximately five minutes and includes almost all frequency bands of the RR signal according to Richardson et al.[10]. Furthermore, for both PRSA indexes $(A C$ and $D C)$, those $[T, s]$ values which showed the best compromise between sensitivity and specificity in discriminating groups and, at the same time, provided a significant p-value, were chosen as the optimum ones. Fig. 2 shows the $A U C$ for $A C$ and $D C$, depending on $T$ and $s$ values, from $24 \mathrm{~h}$ recordings when comparing NRM vs. IDC. The highest values of $A U C$ are located on low $T$ and low $s$ regions, although there are high $A U C$ values for high $T$ values and low $s$ values, as well.

Since $A C[T, s]$ and $D C[T, s]$ responses are nearly symmetric, only $D C$ responses are shown for different analyzed RR series and for simulated signals (Fig. 3 and 4, respectively). 
In Fig. 3, it is shown the behavior of $D C$ as a function of $s$ value for three different values of $T=\{1,5,20\}$. This behavior corresponds to the analysis of 24-hour, awake and sleep periods of one subject of each NRM, LR and HR analyzed groups. As it can be seen in Fig. 3(a), (b) and (c), $D C$ decreases with $s$ when $T=1$. However, when $T$ increases (Fig. 3(d) to (i)), $D C$ initially increases and after decreases (for increasing values of $s$ ), reaching a stationary state. It should be noticed that, in all cases, inflection point occurs approximately for $s=T$; therefore this inflection point is shifted to higher $s$ values when $T$ increases. Given that, from a certain $s$ value, $D C$ indexes for NRM, LR and HR subjects tend to converge, low values of $s$ (and $T$ ) seems to be more suitable for risk stratification, as it has been said previously.

Responses of $D C$ depending on $s$ value for the simulated data are contained in Fig. 4, for $T=\{1,5,20\} . D C$ calculated from GWN series (Fig. 4(a)) shows an inflection point where $s=T$. Previous to the inflection point, $D C$ has an approximately constant behavior and after $D C$ tends to zero. With regard to $D C$ obtained from $A R L F C$ processes (Fig. 4(b)), a damped oscillatory behavior that tends to zero is exhibited. It is observed that values of $D C$ are very similar for $T=1$ and $T=5$, but they are reduced for $T=20 . D C$ calculated from $A R H F c$ processes showed also a certain damped oscillatory behavior that tends to zero (Fig. 4(c)). In this case, results are very little dependent on $T$ value. It can be noticed that the response of these HF simulated data is faster than the response of LF simulation processes.

\section{$3.2 N R M v s . I D C$}

Table II summarizes temporal, spectral and PRSA indexes from 24h-ECG recordings of healthy group and IDC patients. When compared to healthy subjects, the pathological group presented a higher meanRR value ( $p$-value $=0.044)$, but a lower $S D N N$ value ( $p$-value $=0.003$ ). On the other hand, $L F n$ and $L F / H F$ were higher in healthy subjects than in IDC patients. Meanwhile, VLFn was higher in the IDC group ( $p$-value $<0.0005$ ). With regard to PRSA indexes, absolute values of $A C$ and $D C$ were higher in NRM group than in IDC group ( $p$-value $<0.0005$, sen and spe $>60 \%$ ), which means that acceleration capacities increase and deceleration capacities decrease, when risk increases. $A C[1,3]$ and $D C[1,4]$ were the ones that provided the highest sen $(83.3 \%)$ and spe $(74.2 \%$ for $A C$ and $71.0 \%$ for $D C)$ in discriminating between NRM and IDC groups. For both groups, $A C$ and $D C$ were asymmetric ( $p$-value $<0.001$ ). Indexes $A E, D E$ and $A A$ showed significant p-values. However, classification results were not better than those provided by $A C$ and $D C$. In this analysis, $A E$ and $D E$ were higher in the healthy group. Meanwhile, $A A$ was higher in the IDC group.

Moreover, temporal, spectral and PRSA indexes were obtained from awake and sleep periods of healthy and IDC groups (Tables III and IV).

When analyzing awake periods (Table III), the pathological group presented lower value of $S D N N$ than healthy subjects ( $p$ value $=0.0039)$. With regard to spectral domain measures of $\mathrm{HRV}, L F n$ and $L F / H F$ were higher in healthy subjects than in IDC 
185

patients; while $V L F n$ and $H F n$ were higher in the IDC group. However, only $L F / H F$ provided sen and $s p e>60 \%$. Absolute values of $A C$ and $D C$ were higher in NRM group than in IDC group ( $p$-value $<0.0005$ ), as these from 24h-ECG recordings, meaning that $A C$ increase and $D C$ decrease when risk increases. $A C[1,3]$ and $D C[1,3]$ were the ones that provided the highest sen $(90.5 \%$ and $88.1 \%$, respectively) and spe (71\% and 69.4\%, respectively) in discriminating between NRM and IDC groups. In all cases, $A C$ and $D C$ for both groups were asymmetric ( $p$-value $<0.0005)$. In this analysis, $A E$ and $D E$ were higher in the healthy group ( $p$ value $<0.0005$, sen and spe $>60 \%$ ). However, classification results were not better than those provided by $A C$ and $D C$. On the other hand, there were no significant differences between the areas.

Results from sleep periods are included in Table IV. When compared to healthy subjects, the pathological group presented lower values of $S D N N$ and $H F n$ and higher value of $V L F n$. However, none of them provided good classification results (sen and spe $<60 \%$ ). The remaining temporal and spectral measures showed no significant differences between both groups. With regard to PRSA indexes, absolute values of $A C$ and $D C$ were higher in NRM group than in IDC group ( $p$-value $<0.0005$ ), acceleration capacities increase and deceleration capacities decrease. $A E$ and $D E$ were significantly higher in the healthy group, as well. Although these indexes provided high sensitivity, specificity was $<60 \%$. Also in this case, $A C$ and $D C$ for both groups (NRM and IDC) showed asymmetric behavior.

\subsection{LR vs. HR}

Table V sums up temporal, frequency-domain and PRSA indexes from 24h-ECG recordings of LR and HR groups. Both meanRR and $S D N N$ significantly discriminated HR from LR patients (sen and spe $>60 \%$ ). When comparing these two groups of patients, HR participants presented shorter meanRR and lower $S D N N$, compared to LR patients. With regard to spectral domain measures, $L F n$ and $L F / H F$ were higher in the LR group ( $p$-value $<0.05$, sen and spe $>60 \%$ ), while $H F n$ was higher in the HR group ( $p$-value $<0.005$, sen and spe $\geq 60 \%$ ). Regarding to PRSA indexes, absolute values of $A C$ and $D C$ were higher in LR group than in HR group, meaning that $A C$ increase and $D C$ decrease when risk increases. $A C[3,10]$ and $D C[3,10]$ were the ones that provided the best power of classification between LR and HR groups, with sen $=83.3 \%$ and $s p e=80 \%$. The asymmetry was presented between all $A C$ and $D C$ for both groups (from $p$-value $=0.036$ to $p$-value $\leq 0.0005$ ), except for $A C[1,2]$ and $D C[1,2]$ from the HR group. Results of all $A E, D E, A A$ and $D A$, except $A E[1]$ and $D E[1]$, showed significant p-values and provided sen and spe $>60 \%$. However, classification results were not better than those provided by $A C$ and $D C$. In this analysis, $A E$ and $D E$ and also all the areas of PRSA waveforms were higher in LR group.

For the same forty-two patients, all temporal, spectral and PRSA indexes were calculated from awake and sleep periods (Tables VI and VII, respectively). 
In analyzing awake periods (Table VI), the LR patients presented significantly higher meanRR and $S D N N$ values, in the manner of parameters from $24 \mathrm{~h}-\mathrm{ECG}$ recordings. Although both parameters showed significant $p$-value, only meanRR provided sen and spe $>60 \%$. On the other side, the spectral parameters showed no significant statistical differences between HR and LR groups from awake periods. Absolute values of $A C$ and $D C$ were higher in LR group (from $p$-value $=0.0117$ to $p$-value $<0.005$ ), as those from 24h-ECG recordings. $A C$ and $D C$ indexes for $[T, s]=[1,2]$ showed very high sen but $s p e<60 \%$, even though $A U C$ of these indexes was very high. On the other hand, $A C[3,10]$ and $D C[3,10]$ provided better specificity, but at expense of decreasing sensitivity. In this analysis, $A C$ and $D C$ for both groups showed asymmetries (p-value $<0.05)$, except for $A C[1,2]$ and $D C[1,2]$ from the HR group. Results for acceleration and deceleration excursions and PRSA waveforms areas showed significant pvalues, except $A E$ and $D E$ for $T=1$. However, only $D A[1]$ and $A A$ and $D A$ for $T=3$ provided sen and spe $>60 \%$. In this analysis, all these indexes were higher in the LR group.

With regard to temporal parameters from sleep periods (Table VII), LR patients showed a noticeable higher meanRR and $S D N N$ ( $p$-value $<0.001$, sen and spe $>60 \%$ ). In this analysis, $L F n$ was higher in the LR group, while $H F n$ was slightly higher in the HR group. As it can be seen, although both indexes showed significant p-value, only $L F n$ provided good classification results (sen and spe $>60 \%$ ). Absolute values of $A C$ and $D C$ were higher in LR group than in HR group, meaning that $A C$ increases and $D C$ decreases when risk increases. $A C$ and $D C$ for $[T, s]=[1,2]$ and $[T, s]=[3,10]$ provided great classification results between HR and LR participants, with sensitivities of even $91.7 \%$. In almost all cases, $A C$ and $D C$ for both groups were asymmetric (from $p$ value $=0.041$ to $p$-value $\leq 0.0005$ ), except for $A C[3,10]$ and $D C[3,10]$ from the HR group. All excursions and areas of PRSA waveforms showed significant p-values. However, only $A A$ and $D A$ for $T=1$ and $T=3$ provided sen and $s p e>60 \%$. In this analysis, all these indexes were also higher in the LR group.

\section{Discussion}

\subsection{Methodological Aspects}

In this study, the PRSA method proposed by Bauer et al.[13] has been applied to both experimental RR intervals and simulated data. The advantage of this method is that permits to characterize separately acceleration and deceleration processes of heart rate, which are supposed to be related to sympathetic and vagal activities, respectively. The purpose of this study was to analyze acceleration and deceleration processes of beat-to-beat intervals and to try to find significant risk predictors of SCD.

In some cases, it was possible to find values of $[T, s]$ that maximize the statistical classification for both $A C$ and $D C$. However, in other cases, different values of $[T, s]$ were necessary to achieve the best predictive power of $A C[T, s]$ and $D C[T, s]$, according with the concept of asymmetry presented between these two indexes. 
As it has been shown in this paper, responses of $D C$ depending on $s$ value for the GWN simulated data present an inflection point where $s=T$ (Fig. 4(a)). This observation suggests that the inflection point is due to the stochastic behavior of the signal. Since this inflection point is also observed in $D C$ responses obtained from experimental data (Fig. 3), it can be inferred that this PRSA index includes a certain stochastic component. This stochastic behavior seems to contradict the statement of that PRSA technique eliminates non-periodic components (artifacts, noise...) from complex signals[13,22].

In Fig. 3(a)-(c), it can be seen a fast response of $D C$ with respect to $s$ and similar to that obtained from $A R H F C$ simulated processes (Fig. 4(c)). In Bauer et al.[13], it is said that the increase of temporal scale $T$ sets an upper frequency limit for periodicities that can be detected by PRSA (a kind of low-pass filter). According to this, in the case where $T=1$, all frequencies of the signal are taken into account. From these observations, we can conclude that the HF prevails in $D C$, when $T=1$. Moreover, the damped oscillatory behavior of $A R H F c$ is only shown in the analysis of sleep periods, which indicates that HF is more noticeable during sleep than during awake periods.

However, when $T$ value increases (Fig. 3(d)-(i)), the response of $D C$ calculated from experimental data is slower. This response is more similar to the behavior of the $A R L F c$ simulated processes (Fig. 4(b)). This fact seems to be in concordance with Bauer's statement about the pass-filter effect of increasing $T$ value. This equalizes PRSA responses more in HR group than LR group and more in awake periods than in sleep periods, indicating an increase of high frequencies from awake to sleep periods.

\section{$4.3 N R M$ vs.IDC}

From 24h-ECG recordings, it was found that some $A C, D C, A E$ and $D E$ discriminated IDC from NRM subjects better than traditional temporal and frequency measures. Although $A C[1,2]$ provided the best sensitivity, other couples of $[T, s]$, like [1,3] and $[1,4]$, provided $A C$ and $D C$ indexes, which showed a better spe $(>70 \%)$ maintaining a very good sen (>80\%) in discriminating NRM from IDC subjects. Statistical classification results from $A E$ and $D E$ were not as good as those from $A C$ and $D C$. In addition, these PRSA indexes provided better $A U C$ than all temporal and spectral measures.

It should be noticed that, in all selected PRSA indexes, $T$ value was equal to 1 . That means that all periodicities are considered, since increasing $T$ is like establishing a kind of low-past filter. This optimal $T$ value coincides with the value established in the work of Kantelhardt et al. $[14]$ as the best $[T, s]$ values for prediction of mortality after heart attack, $[T, s]=[1,2]$. However, a slightly increase of the wavelet scale $s$ permits to improve predictive results in this analysis (NRM vs.IDC), as it is shown in Table II.

Bauer et al.[22] concluded that $D C$ is more powerful predictor of mortality after myocardial infarction than $A C$. Conversely, we have found that, between IDC and control subjects, both capacities provide similar predictive power (Table II). Results from 
274 Kisohara et al.[23] also showed similar predictive values for $A C$ and $D C$, when predicting mortality after acute myocardial infarction

Bauer hypothesis affirms that $A C$ and $D C$ could provide non-invasive measurements of cardiac sympathetic and vagal modulations, respectively [22]. Evidence of experimental and clinical studies [1,24] indicates that a fall of vagal activity increases the risk of death. Results obtained here for $D C$ are in agreement with those evidences, since $D C$ is reduced in IDC patients. However, unlike the results from Bauer et al.[22], results obtained in the present study showed also a significant increase of $A C$ in pathological subjects. It is believed that sympathetic activity is increased in pathological conditions[21]. Thus, results of the present study are consistent also with this observation, indicating that $A C$ parameter could reflect the sympathetic activity, as Bauer stated.

Moreover, it has been verified that $A C$ and $D C$ are correlated with $S D N N$. Thus, absolute values of $A C$ and $D C$ reflect also variability of cardiac rhythm. Furthermore, increasing absolute values of PRSA indexes could also reflect a lower ANS regularity. In their research, Guzik et al.[25] used a distribution function of deceleration and acceleration that was similar to $S D N N^{2}$.

It is known that under normal conditions, both branches of the ANS are tonically active when regulating cardiac activity with a certain dominance of vagal regulation[26]. In general, absolute values of $D C$ and $A C$ are asymmetric, with $D C$ slightly higher than $A C$. Since $D C$ is believed to be related to vagal activity, these results are consistent, for healthy subjects, with physiological observations. These asymmetry results are also consistent with results from previous studies[25,27,28], where it was proved that the pattern of heart rate during acceleration is different to the pattern of deceleration in normal subjects. Cysarz et al.[29] stated that sympathetic modulations are slower than parasympathetic modulations captured respectively by acceleration and deceleration changes of the heart rate of healthy subjects. In our study, some asymmetries have been found between acceleration and deceleration patterns of IDC patients, as well.

Besides analyzing 24h-ECG recordings from healthy and IDC patients, awake and sleep periods were analyzed. Statistical results showed that considering only awake periods, predictive power of $A C$ and $D C$ was similar (with little increasing in sen) to that obtained from 24h-ECG recordings. Also $A E$ and $D E$ calculated from awake periods showed similar predictive results than those from $24 \mathrm{~h}-\mathrm{ECG}$ recordings. Nevertheless, analysis of sleep periods provided lower specificities $(<60 \%)$ for $A C, D C, A E$ and $D E$ than those from $24 \mathrm{~h}$ recordings and awake periods. In any case, $A A$ and $D A$ were not able to discriminate between NRM and IDC subjects.

When analyzing awake periods, absolute $A C$ and $D C$ values for both groups were slightly lower than those calculated from 24h recordings. However, these PRSA indexes increased their absolute values when they were calculated from sleep periods. This agrees with results from Carvajal et al.[30], which showed a higher complexity during the night. 
$A C$ values were lower in sleep periods than in awake periods, while $D C$ values were higher in sleep periods, indicating a decreasing sympathetic activity and an increasing vagal activity during sleep, respectively. Also, certain asymmetry exists between these $|A C|$ and $|D C|$ values, being the deceleration capacity higher. As it has been said before, deceleration capacity is supposed to be related to vagal activity, which is predominant during rest. The fact that $D C$ was higher than $A C$ during sleep periods agrees with that evidence. On the other side, regarding to awake periods, also a certain prevalence of vagal activity $(|D C|>|A C|)$ was shown, which is consistent with the knowledge of both systems are in equilibrium in normal conditions, with certain predominance of vagal activity[26].

\subsection{LR vs. HR}

From 24h-ECG recordings, it has been found that $A C[1,2], D C[1,2]$ and $S D N N$ discriminated HR patients from LR patients with high sensitivity $(s e n=83.3 \%)$ and specificity $(s p e=70 \%)$. For risk stratification of these patients, the increase of $T$ and $s$ ([T,s] $=[3,10])$ for $A C$ and $D C$ provided improvement in spe to $80 \%$. Most of the $A E, D E$, $A A$ and $D A$ provided slightly lower classification results when compared with $A C$ and $D C$. PRSA indexes were almost as good as $S D N N$ when discriminating HR from LR patients. However, spectral measures showed lower predictive results.

In the present analysis, $\mathrm{HR}$ patients showed an increasing $A C$ and decreasing $D C$ compared with LR patients. Considering that $A C$ is related to sympathetic modulations and $D C$ to vagal regulation, these results agree with experimental evidence of that cardiac risk is increased when sympathetic activity increases and/or vagal activity decreases[1,10,24]. As it has been previously shown, absolute values of $A C$ and $D C$ were higher in LR patients than in HR. This can be interpreted as a lower regularity of the ANS in LR group. Similar results in post-infarction patients with increased risk of mortality have been reported by other researches[28], where deceleration was reduced during a two-year follow-up. This observation is in agreement with physiological evidences, since reduced HRV is associated with higher cardiac risk.

Temporal and spectral measures and PRSA indexes were also analyzed for awake and sleep periods. Statistical results showed that, considering only awake periods, predictive power of $A C$ and $D C$ were lower than those obtained from $24 \mathrm{~h}$-ECG recordings. Some of the PRSA waveform areas from awake periods provided similar classification results to those from $24 \mathrm{~h}-\mathrm{ECG}$ recordings. On the other hand, analysis of sleep periods provided better sensitivity, but lower specificity for $A C$ and $D C$ than those from $24 \mathrm{~h}-\mathrm{ECG}$ recordings and awake periods.

\section{Conclusions}

The aim of the present explorative study was to identify essential aspects of the acceleration and deceleration cardiac process implicated in the heterogeneous etiology of IDC disease. From the analysis of PRSA responses, it was deduced that the inflection 
334 point observed in the PRSA curves reflects a certain stochastic behavior of the signals. In addition, it can be concluded that the upper frequencies of the RR series prevail in $D C$, when $T=1$, and was more noticeable during sleep than during awake periods. However, when $T$ value increases, the response of $D C$ calculated from experimental data was slower and more similar to the behavior of the low frequency oscillations.

$A C$ and $D C$ indexes, mainly for low values of $T$ and $s$, provided significant discrimination between different risk of SCD patients, in some cases improving results from traditional temporal and spectral HRV measures. It can be also stated that $A C$ and $D C$ indexes have permitted to obtain better cardiac risk stratification than using autonomic information flow as in Palacios et al.[31], analyzing similar myocardial pathology.

Results obtained from PRSA analysis showed a higher $A C$ (which reflects sympathetic activity) and a lower $D C$ (which reflects vagal activity) in patients with higher risk of cardiac death, which agrees with the fact that the risk of cardiac death is increased by a growth in sympathetic activity and/or a fall in vagal activity.

Finally, new applications over different pathological populations are necessary to better understand whether $A C$ works slightly better than $D C$ or this performance is only presented in the analysis of RR series of IDC patients. 
366
Journal: MEDICAL ENGINEERING \& PHYSICS

\section{Title of Paper:}

Acceleration and Deceleration Capacities for Heart Rate Analysis and Risk Stratification in Idiopathic Dilated Cardiomyopathy

\section{Declarations}

The following additional information is required for submission. Please note that failure to respond to these questions/statements will mean your submission will be returned to you. If you have nothing to declare in any of these categories then this should be stated.

\section{Conflict of interest}

All authors must disclose any financial and personal relationships with other people or organisations that could inappropriately influence (bias) their work. Examples of potential conflicts of interest include employment, consultancies, stock ownership, honoraria, paid expert testimony, patent applications/registrations, and grants or other funding.

\section{Ethical Approval}

Work on human beings that is submitted to Medical Engineering \& Physics should comply with the principles laid down in the Declaration of Helsinki; Recommendations guiding physicians in biomedical research involving human subjects. Adopted by the 18th World Medical Assembly, Helsinki, Finland, June 1964, amended by the 29th World Medical Assembly, Tokyo, Japan, October 1975, the 35th World Medical Assembly, Venice, Italy, October 1983, and the 41st World Medical Assembly, Hong Kong, September 1989. You should include information as to whether the work has been approved by the appropriate ethical committees related to the institution(s) in which it was performed and that subjects gave informed consent to the work.

\section{Competing Interests}

None declared

Please state any sources of funding for your research

None

DOES YOUR STUDY INVOLVE HUMAN SUBJECTS? Please cross out whichever is not applicable.

Yes

No

If your study involves human subjects you MUST have obtained ethical approval.

Please state whether Ethical Approval was given, by whom and the relevant Judgement's reference number The study protocol was approved by institutional Investigation Committees and all subjects signed informed consent.

This information must also be inserted into your manuscript under the acknowledgements section prior to the References. 
412

413

414

415

416

417

418

419

420

421

422

423

424

425

426

427

428

429

430

431

432

433

434

435

436

437

438

439

440

441

442

443

444

445

446

447

448

449

450

451

452

453

454

455

456

457

458

459

460

461

462

463

464

465

\section{Acknowledgment}

This work was supported within the framework of the CICYT grant TEC2010-20886 from the Spanish Government. CIBER of Bioengineering, Biomaterials and Nanomedicine is an initiative of ISCIII.

(1)

(1)

(1)

(1)

(6)




\section{References}

[1] Lown B, Verrier RL. Neural activity and ventricular fibrillation. New England Journal Medical 1976;294:1165-70.

[2] Algra A, Tijssen JGP, Roelandt JRTC, Pool J, Lubsen J. Heart rate variability from 24-hour electrocardiography and the 2year risk for sudden death. Circulation 1993;88:180-5.

[3] Moraru L, Tong S, Malhotra A, Geocadin R, Thakor N, Bezerianos A. Investigation of the effects of ischemic preconditioning on the HRV response to transient global ischemia using linear and nonlinear methods. Med Eng Phys $2005 ; 27: 465-73$.

[4] Baumert M, Baier V, Haueisen J, Wessel N, Myerfeldt U, Schirdewan A, Voss A. Forecasting of life threatening arrhythmias using the compression entropy of heart rate. Meth Information Med 2004;43:202-6.

[5] Hoyer D, Friedrich H, Zwiener U, Pompe B, Baranowski R, Werdan K, Muller-Werdan U, Schmidt H. Prognostic impact of autonomic information flow in multiple organ dysfunction syndrome patients. Int J Cardiol 2006;108:359-69.

[6] Todder D., Bersudsky Y., Cohen H. Nonlinear analysis of RR interval in euthymic bipolar disorder. Auton Neurosci $2005 ; 117: 27-31$.

[7] Voss A, Baier V, Schulz S, Bar KJ. Linear and nonlinear methods for analyses of cardiovascular variability in bipolar disorders. Bipolar Disord 2006;8:441-52.

[8] Binkley PF, Nunziata E, Haas GJ, Nelson SD, Cody RJ. Parasympathetic withdrawal is an integral component of autonomic imbalance in congestive heart failure: demonstration in human subjects and verification in a paced canine model of ventricular failure. J Am Coll Cardiol 1991;18(2):464-72.

[9] Floras JS. Sympathetic nervous system activation in human heart failure clinical implications of an updated model. Journal of the American College of Cardiology 2009;54(5):375-85.

[10] Richardson P et al. Report of the 1995 World Health Organization/International Society and Federation of Cardiology Task Force on the definition and classification of cardiomyopathies. Circulation 1996;93:841-2.

[11] Mateo J, Laguna P. Improved heart rate variability signal analysis from the beat occurrence times according to the IPFM model. IEEE Transactions on Biomedical Engineering 2000;47:985-96.

[12] Goren Y, Davrath LR, Pinhas I, Toledo E, Akselrod S. Individual time-dependent spectral boundaries for improved accuracy analysis of heart rate variability. IEEE on Biomedical Engineering 2006;53:35-42.

[13] Bauer A, Kantelhardt JW, Bunde A, Barthel P, Schneider R, Malik M, Shmidt G. Phase rectified signal averaging detects quasi-periodicities in nonstationary data. Physica A 2006;364:423-34. 
[14] Kantelhardt JW, Bauer A, Schumann AY, Barthel P, Schneider R, Malik M, Schmidt G. Phase-rectified signal averaging for the detection of quasi-periodicities and the prediction of cardiovascular risk. Chaos 2007;17:015112.

[15] Maron BJ, Towbin JA, Thiene G, Antzelevitch C, Corrado D, Arnett D, Moss AJ, Seidman CE, Young JB. Contemporary definitions and classification of the cardiomyopathies. An American Heart Association Scientific Statement from the Council on Clinical Cardiology, Heart Failure and Transplantation Committee; Quality of Care and Outcomes Research and Functional Genomics and Translational Biology Interdisciplinary Working Groups; and Council on Epidemiology and Prevention. Circulation 2006;113:1807-16.

[16] IDEAL Database: Intercity Digital ECG Alliance organized by University of Rochester Medical Center, Rochester, USA, 1997.

[17] Martinez JP, Almeida R, Olmos S, Rocha AP, Laguna P. A wavelet-based ECG delineator: evaluation on standard databases. IEEE Transactions on Biomedical Engineering 2004;51:570-81.

[18] Valencia JF, Vallverdú M, Schroeder R, Voss A, Vázquez R, Bayés de Luna A, Caminal P. Complexity of the short-term heart-rate variability. IEEE Eng Med Biol 2009;28:72-8.

[19] Clifford GD, McSharry PE, Tarassenko L. Characterizing artefact in the normal human 24-hour RR time series to aid identification and artificial replication of circadian variations in human beat to beat heart rate using a simple threshold. Computers in Cardiology 2002;29:129.

[20] Wessel N, Voss A, Kurths J, Schirdewan A, Hnatkova K, Malik M. Evaluation of renormalized entropy for risk stratification using heart rate variability data. Med Biol Eng Comput 2000;38:680.

[21] Task Force of the European Society of Cardiology and the North American Society of Pacing and Electrophysiology on Heart Rate Variability. European Heart Journal 1996;17:354-81.

[22] Bauer A, Kantelhardt JW, Barthel P, Schneider R, Makikallio T, Ulm K. Deceleration capacity of heart rate as a predictor of mortality after myocardial infarction: cohort study. Lancet 2006;367:1674-81.

[23] Kisohara M, Stein PK, Yoshida Y, Suzuki M. Multi-scale heart rate dynamics detected by phase-rectified signal averaging predicts mortality after acute myocardial infarction. European Society of Cardiology 2012; doi:10.1093/europace/eus409.

[24] Lahiri MK, Kannankeril PJ, Goldberger JJ. Assessment of autonomic function in cardiovascular disease: Physiological basis and prognostic implications. J Am Coll Cardiol 2008;51:1725-33.

[25] Guzik P, Piskorski J, Krauze T, Wykretowicz A, Wysocki H. Heart rate asymmetry by Poincare plots of RR intervals. Biomed Tech 2006;51:272-5.

[26] Hainsworth R. The control and physiological importance of heart rate. In: Malik M, Camm AJ. editors. Heart rate variability. Armonk, NY: Future Publ Comp Inc 1995, p. 3-19. 
[27] Porta A, Casali KR, Casali AG, Gnecchi-Ruscone T, Tobaldini E, Montano N, Lange S, Geue D, Cysarz D, Van Leeuwen P. Temporal asymmetries of short-term heart period variability are linked to autonomic regulation. Am J Physiol Regul Integr Comp Physiol 2008;295:R550-7.

[28] Piskorski J, Guzik P. Structure of heart rate asymmetry: deceleration and acceleration runs. Physiol Meas 2011;32:1-13.

[29] Cysarz D, Porta A, Montano N, Leeuwen PV, Kurths J, and Wessel N. Quantifying heart rate dynamics using different approaches of symbolic dynamics. The European Physical Journal Special Topics 2013;222:487-500.

[30] Carvajal R, Wessel N, Vallverdú M, Caminal P, Voss A. Correlation dimension analysis of heart rate variability in patients with dilated cardiomyopathy. Computer Methods and Programs in Biomedicine 2005;2405:1-8.

[31] Palacios M, Friedrich H, Götze C, Vallverdú M, Bayes de Luna A, Caminal P, Hoyer D. Changes of autonomic information flow due to idiopathic dilated cardiomyopathy. Physiological Measurement 2007;28(6):677-88. 


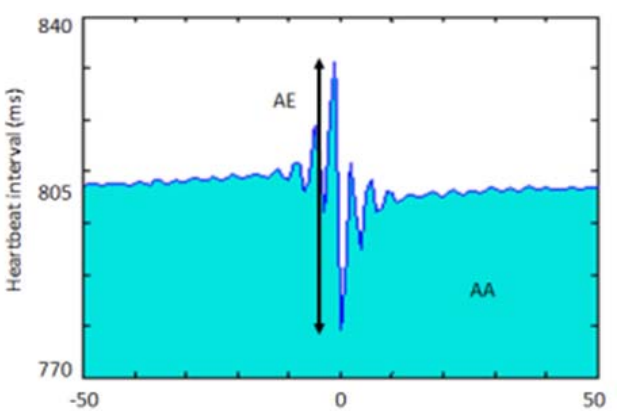

(a)

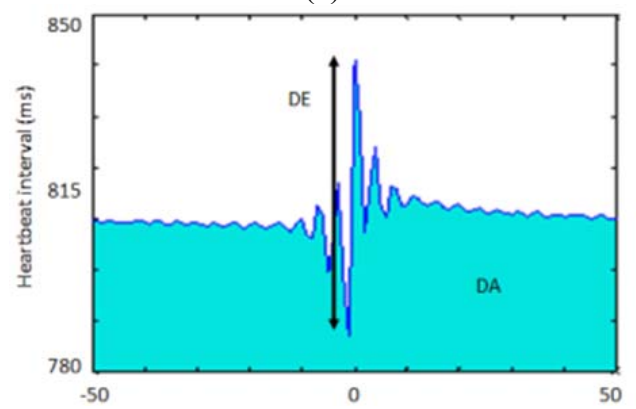

(b)

Fig. 1. (a) Definition of range of acceleration excursion $(A E)$ and the area under the acceleration PRSA waveform $(A A)$; (b) Definition of range of deceleration excursion $(D E)$ and the area under the deceleration PRSA waveform $(D A)$.
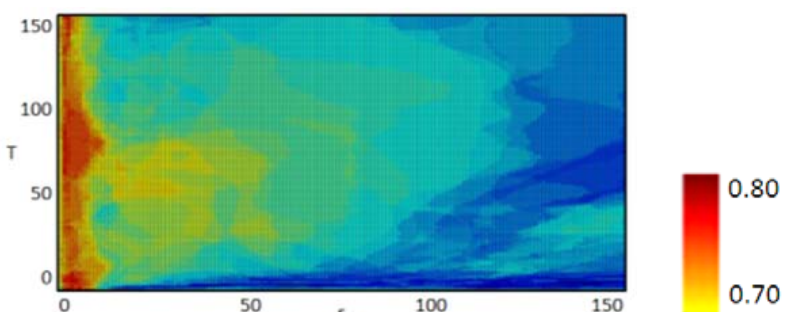

(a)

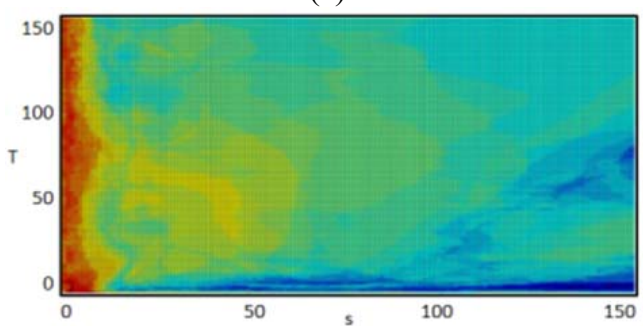

Fig. 2. Color-scale plot of the $A U C$ (area under the ROC curve) for $A C$ (a) and $D C$ (b) depending on $T$ and $s$ values for $24 \mathrm{~h}$ recordings when comparing NRM vs. IDC. 


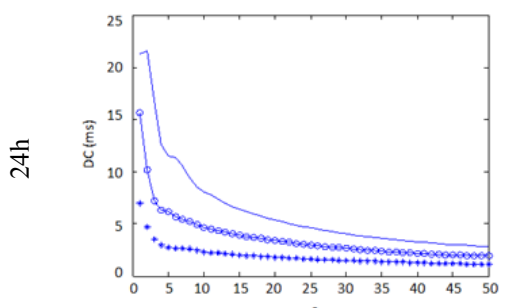

(a)

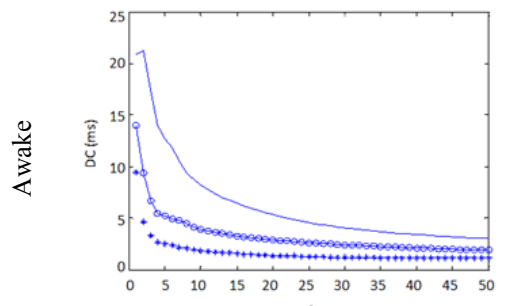

(b)

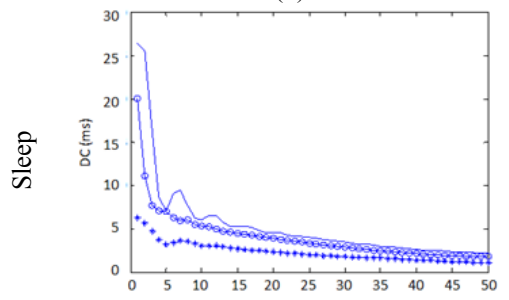

(c)

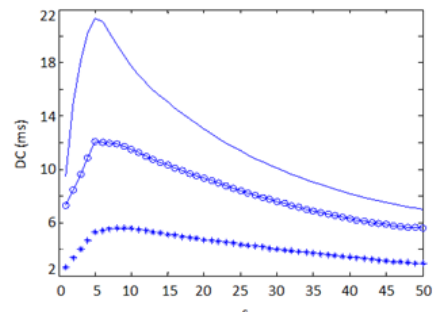

(d)

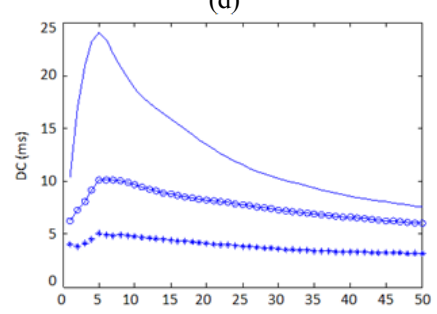

(e)

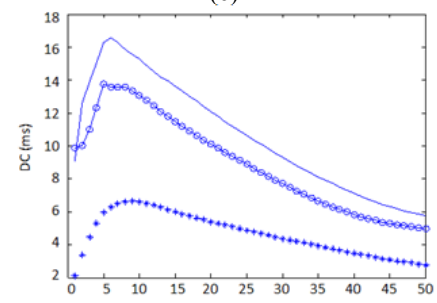

(f)

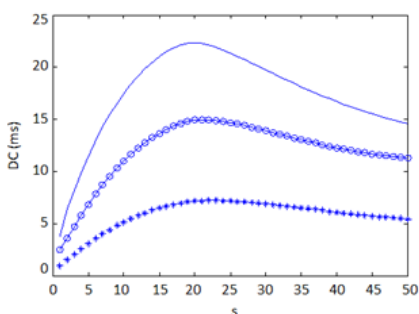

(g)

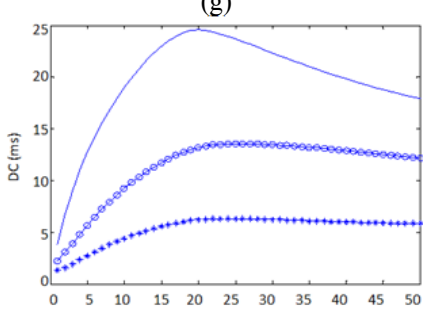

(h)

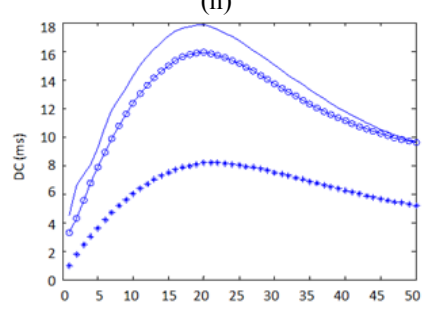

(i)

Fig. 3. Behavior of $D C$ depending on $s$ value for $T=1$ ((a), (b), (c)), $T=5((\mathrm{~d}),(\mathrm{e}),(\mathrm{f})), T=20((\mathrm{~g}),(\mathrm{h}),(\mathrm{i}))$ from $24 \mathrm{~h}$ recordings, awake periods and sleep periods, respectively. Solid line corresponds to a NRM subject, dot line to a LR patient and asterisks to a HR patient.

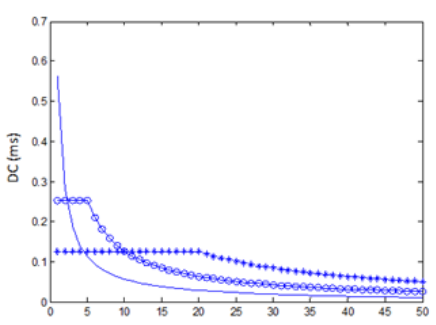

(a)

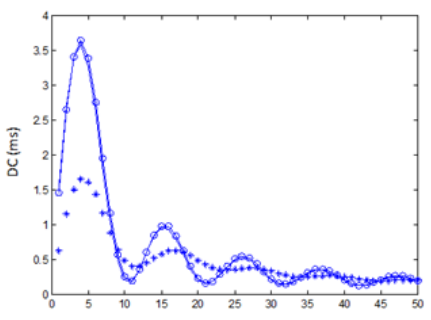

(b)

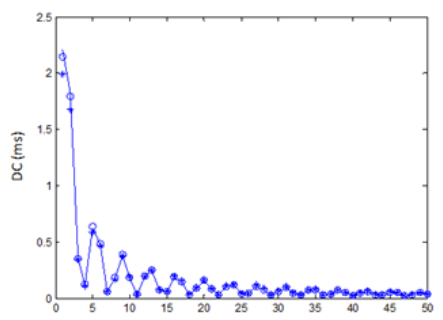

(c)

Fig. 4. Behavior of $D C$ depending on $s$ value for $T=1$ (solid line), $T=5$ (dot line), $T=20$ (asterisks) from different simulated series: (a) GWN, (b) $A R L F c$ and (c) $A R H F c$. 
TABLE II

RR INTERVALS OF 24H-ECG RECORDINGS FROM HEALTHY (NRM) AND IDC SUBJECTS

\begin{tabular}{llllll}
\hline \hline & $\begin{array}{l}\text { NRM } \\
\text { mean } \pm \text { std }\end{array}$ & $\begin{array}{l}\text { IDC } \\
\text { mean } \pm \text { std }\end{array}$ & $\begin{array}{l}\text { Sen } \\
(\%)\end{array}$ & $\begin{array}{l}\text { Spe } \\
(\%)\end{array}$ & AUC \\
\hline$N$ (subjs. $)$ & 62 & 42 & & & \\
meanRR & $773.6 \pm 98.8$ & $783.7 \pm 103.9 *$ & 28.6 & 77.4 & 0.558 \\
$S D N N$ & $146.7 \pm 49.2$ & $118.8 \pm 46.5 * *$ & 71.4 & 58.1 & 0.672 \\
\hline$V L F n$ & $31.59 \pm 4.24$ & $34.32 \pm 6.47 \ddagger$ & 52.4 & 82.3 & 0.668 \\
$L F n$ & $48.25 \pm 7.08$ & $41.31 \pm 8.82 \dagger$ & 54.8 & 74.2 & 0.711 \\
$H F n$ & $42.65 \pm 5.96$ & $44.37 \pm 5.56 \dagger$ & - & - & - \\
$L F / H F$ & $1.23 \pm 0.38$ & $1.01 \pm 0.34 *$ & 61.9 & 45.2 & 0.652 \\
\hline$A C[1,2]$ & $-12.88 \pm 4.78$ & $-6.97 \pm 3.17 \dagger$ & 85.7 & 67.7 & 0.844 \\
$D C[1,2]$ & $13.32 \pm 4.86$ & $7.38 \pm 3.46 \dagger$ & 78.6 & 66.1 & 0.834 \\
$A C[1,3]$ & $-10.60 \pm 3.23$ & $-5.78 \pm 2.61 \dagger$ & 83.3 & 74.2 & 0.871 \\
$D C[1,3]$ & $11.09 \pm 3.46$ & $6.15 \pm 2.82 \ddagger$ & 81.0 & 71.0 & 0.858 \\
$A C[1,4]$ & $-9.54 \pm 3.05$ & $-5.23 \pm 2.99 \ddagger$ & 83.3 & 72.6 & 0.854 \\
$D C[1,4]$ & $10.05 \pm 3.27$ & $5.58 \pm 2.79 \dagger$ & 83.3 & 71.0 & 0.848 \\
\hline$A E[1]$ & $30.76 \pm 12.75$ & $21.99 \pm 13.35 \ddagger$ & 76.2 & 61.3 & 0.745 \\
$D E[1]$ & $31.94 \pm 12.79$ & $23.16 \pm 14.54 \dagger$ & 73.8 & 67.7 & 0.741 \\
$A A[1]^{*} 10^{4}$ & $23.30 \pm 3.00$ & $23.60 \pm 3.13 *$ & 31.0 & 75.8 & 0.560 \\
$D A[1]^{*} 10^{4}$ & $23.36 \pm 2.94$ & $23.62 \pm 3.13 \dagger$ & - & - & -
\end{tabular}

meanRR, SDNN, AC,DC,AE and DE expressed in ms. VLFn, LFn and $H F n$ expressed in n.u. $A A$ and $D A$ expressed in area units. tp-value $<0.0005,{ }^{*}$ p-value $<0.005,{ }^{*}$ p-value $<0.05, \dagger p$-value $=$ n.s. 
643

644

645

646

647

648

649

650

651

652

653

654

655

656 657
TABLE III

RR INTERVALS OF AWAKE PERIODS OF ECG RECORDINGS FROM HEALTHY (NRM) AND IDC SUBJECTS

\begin{tabular}{llllll}
\hline \hline & $\begin{array}{l}\text { NRM } \\
\text { mean } \pm \text { std }\end{array}$ & $\begin{array}{l}\text { IDC } \\
\text { mean } \pm \text { std }\end{array}$ & $\begin{array}{l}\text { Sen } \\
(\%)\end{array}$ & $\begin{array}{l}\text { Spe } \\
(\%)\end{array}$ & AUC \\
\hline$N($ subjs. $)$ & 62 & 42 & & & \\
$m e a n R R$ & $700.8 \pm 93.2$ & $721.9 \pm 96.3 \dagger$ & - & - & - \\
$S D N N$ & $85.8 \pm 26.4$ & $73.0 \pm 30.4 * *$ & 57.1 & 59.7 & 0.661 \\
\hline$V L F n$ & $32.07 \pm 4.39$ & $34.85 \pm 6.91 \ddagger$ & 57.1 & 75.8 & 0.668 \\
$L F n$ & $51.08 \pm 7.44$ & $41.68 \pm 11.01 \dagger$ & 57.1 & 80.7 & 0.753 \\
$H F n$ & $39.59 \pm 6.05$ & $42.96 \pm 6.65 *$ & 50.0 & 69.4 & 0.648 \\
$L F / H F$ & $1.38 \pm 0.42$ & $1.07 \pm 0.45 \dagger$ & 61.9 & 69.4 & 0.589 \\
\hline$A C[1,2]$ & $-10.98 \pm 4.72$ & $-5.88 \pm 3.18 \dagger$ & 83.3 & 62.9 & 0.834 \\
$D C[1,2]$ & $11.42 \pm 4.53$ & $6.32 \pm 3.51 \dagger$ & 81.0 & 66.1 & 0.825 \\
$A C[1,3]$ & $-10.23 \pm 3.79$ & $-5.06 \pm 2.76 \dagger$ & 90.5 & 71.0 & 0.854 \\
$D C[1,3]$ & $10.82 \pm 3.98$ & $5.49 \pm 3.04 \dagger$ & 88.1 & 69.4 & 0.848 \\
\hline$A E[1]$ & $27.34 \pm 12.04$ & $19.61 \pm 12.67 \dagger$ & 78.6 & 67.7 & 0.733 \\
$D E[1]$ & $28.71 \pm 11.73$ & $20.83 \pm 13.84 \dagger$ & 76.2 & 64.5 & 0.734 \\
$A A[1]^{*} 10^{4}$ & $21.00 \pm 2.77$ & $21.65 \pm 2.87 \dagger$ & - & - & - \\
$D A[1]^{*} 10^{4}$ & $21.07 \pm 2.80$ & $21.66 \pm 2.86 \dagger$ & - & - & - \\
\hline \hline
\end{tabular}

meanR $\overline{\overline{R, S D N N, A C, D C, A E \text { and } D E \text { expressed in ms. } V L F n, L F n \text { and } H F}}$ expressed in n.u. $A A$ and $D A$ expressed in area units. $\pitchfork$ p-value $<0.0005,{ }^{*}$ p-value $<0.005,{ }^{*}$ p-value $<0.05, \dagger$-value $=$ n.s.

TABLE IV

RR INTERVALS OF SLEEP PERIODS OF ECG RECORDINGS FROM HEALTHY (NRM) AND IDC SUBJECTS

\begin{tabular}{llllll}
\hline \hline & $\begin{array}{l}\text { NRM } \\
\text { mean } \pm \text { std }\end{array}$ & $\begin{array}{l}\text { IDC } \\
\text { mean } \pm \text { std }\end{array}$ & $\begin{array}{l}\text { Sen } \\
(\%)\end{array}$ & $\begin{array}{l}\text { Spe } \\
(\%)\end{array}$ & $A U C$ \\
\hline$N$ (subjs.) & 62 & 42 & & & \\
$m e a n R R$ & $953.6 \pm 158.0$ & $907.9 \pm 148.8 \dagger$ & - & - & - \\
$S D N N$ & $97.0 \pm 34.4$ & $82.8 \pm 40.3 *$ & 45.2 & 62.9 & 0.631 \\
\hline$V L F n$ & $29.51 \pm 5.65$ & $32.94 \pm 6.96 \dagger$ & 52.4 & 80.7 & 0.671 \\
$L F n$ & $44.90 \pm 8.53$ & $41.51 \pm 8.90 \dagger$ & - & - & - \\
$H F n$ & $46.45 \pm 7.70$ & $45.43 \pm 6.09^{*}$ & 71.4 & 46.8 & 0.564 \\
$L F / H F$ & $1.06 \pm 0.43$ & $0.99 \pm 0.34 \dagger$ & - & - & - \\
\hline$A C[1,2]$ & $-17.36 \pm 7.86$ & $-9.40 \pm 4.71 \dagger$ & 83.3 & 56.5 & 0.806 \\
$D C[1,2]$ & $18.11 \pm 8.80$ & $9.88 \pm 5.16 \dagger$ & 85.7 & 53.2 & 0.802 \\
\hline$A E[1]$ & $43.57 \pm 23.59$ & $28.59 \pm 16.39 \S$ & 85.7 & 45.2 & 0.716 \\
$D E[1]$ & $45.73 \pm 26.70$ & $30.01 \pm 17.55^{*}$ & 90.5 & 37.1 & 0.700 \\
$A A[1]^{*} 10^{4}$ & $28.54 \pm 4.74$ & $27.19 \pm 4.46 \dagger$ & - & - & - \\
$D A[1]^{*} 10^{4}$ & $28.57 \pm 4.72$ & $27.18 \pm 4.44 \dagger$ & - & - & - \\
\hline \hline
\end{tabular}

meanRR, SDNN, $A C, D C, A E$ and $D E$ expressed in ms. VLFn, $L F n$ and $H F n$ expressed in n.u. $A A$ and $D A$ expressed in area units. $\$$ p-value $<0.0005, \S \mathrm{p}$-value $<0.001,{ }^{*} \mathrm{p}$-value $<0.05, \dagger \mathrm{p}$-value $=\mathrm{n}$.s. 
670

671

672

673

674

675

676

677

678

679

680

681

682

683
TABLE V

RR INTERVALS OF 24H-ECG RECORDINGS FROM LR AND HR PATIENTS

\begin{tabular}{llllll} 
& $\begin{array}{l}\text { LR } \\
\text { mean } \pm \text { std }\end{array}$ & $\begin{array}{l}\text { HR } \\
\text { mean } \pm \text { std }\end{array}$ & $\begin{array}{l}\text { Sen } \\
(\%)\end{array}$ & $\begin{array}{l}\text { Spe } \\
(\%)\end{array}$ & $A U C$ \\
\hline$N$ (subjs.) & 30 & 12 & & & \\
meanRR & $816.0 \pm 94.9$ & $702.9 \pm 80.6 \S$ & 66.7 & 76.7 & 0.839 \\
$S D N N$ & $132.7 \pm 46.4$ & $84.0 \pm 23.7 \dagger$ & 83.3 & 70.0 & 0.861 \\
\hline$V L F n$ & $34.04 \pm 7.12$ & $35.05 \pm 4.66 \dagger$ & - & - & - \\
$L F n$ & $42.40 \pm 9.80$ & $38.58 \pm 5.07^{*}$ & 75.0 & 70.0 & 0.639 \\
$H F n$ & $44.22 \pm 6.22$ & $44.74 \pm 3.63^{* *}$ & 66.7 & 60.0 & 0.558 \\
$L F / H F$ & $1.05 \pm 0.37$ & $0.91 \pm 0.20^{*}$ & 75.0 & 63.3 & 0.586 \\
\hline$A C[1,2]$ & $-8.00 \pm 3.06$ & $-4.41 \pm 1.64 \dagger$ & 83.3 & 70.0 & 0.858 \\
$D C[1,2]$ & $8.52 \pm 3.35$ & $4.53 \pm 1.56 \dagger$ & 83.3 & 70.0 & 0.861 \\
$A C[3,10]$ & $-8.45 \pm 2.57$ & $-5.49 \pm 2.10^{* *}$ & 83.3 & 80.0 & 0.819 \\
$D C[3,10]$ & $8.98 \pm 2.82$ & $5.78 \pm 2.31^{* *}$ & 83.3 & 80.0 & 0.811 \\
\hline$A E[1]$ & $24.64 \pm 14.85$ & $15.36 \pm 4.02 \dagger$ & - & - & - \\
$D E[1]$ & $26.17 \pm 16.11$ & $15.65 \pm 4.28 \dagger$ & - & - & - \\
$A A[1]^{*} 10^{4}$ & $24.58 \pm 2.86$ & $21.15 \pm 2.41 \S$ & 66.7 & 76.7 & 0.839 \\
$D A[1]^{*} 10^{4}$ & $24.61 \pm 2.86$ & $21.16 \pm 2.40 \S$ & 66.7 & 80.0 & 0.847 \\
$A E[3]$ & $25.97 \pm 9.99$ & $16.09 \pm 5.71^{* *}$ & 83.3 & 63.3 & 0.817 \\
$D E[3]$ & $27.57 \pm 10.77$ & $16.78 \pm 6.02^{* *}$ & 83.3 & 63.3 & 0.828 \\
$A A[3]^{*} 10^{4}$ & $24.43 \pm 2.85$ & $21.07 \pm 2.39 \S$ & 66.7 & 76.7 & 0.836 \\
$D A[3]^{*} 10^{4}$ & $24.48 \pm 2.82$ & $21.10 \pm 2.42 \S$ & 66.7 & 76.7 & 0.836 \\
\hline \hline
\end{tabular}

meanR $\overline{\overline{R, S D N N, A C, D C, A E \text { and } D E \text { expressed in ms. VLFn, } L F n \text { and } H F}}$ expressed in n.u. $A A$ and $D A$ expressed in area units. $\$ \mathrm{p}$-value $<0.0005, \S \mathrm{p}$-value $<0.001, * * \mathrm{p}$-value $<0.005, * \mathrm{p}$-value $<0.05$, $\dagger \mathrm{p}$-value $=$ n.s.

\section{TABLE VI}

RR INTERVALS OF AWAKe PERIODS OF ECG RECORDINGS FROM LR AND HR PATIENTS

\begin{tabular}{llllll}
\hline \hline & $\begin{array}{l}\text { LR } \\
\text { mean } \pm \text { std }\end{array}$ & $\begin{array}{l}\text { HR } \\
\text { mean } \pm \text { std }\end{array}$ & $\begin{array}{l}\text { Sen } \\
(\%)\end{array}$ & $\begin{array}{l}\text { Spe } \\
(\%)\end{array}$ & $A U C$ \\
\hline$N($ subjs.) & 30 & 12 & & & \\
$m e a n R R$ & $749.5 \pm 94.4$ & $652.8 \pm 61.3^{* *}$ & 83.3 & 60.0 & 0.839 \\
$S D N N$ & $80.1 \pm 31.5$ & $55.2 \pm 18.7^{*}$ & 83.3 & 50.0 & 0.767 \\
\hline$V L F n$ & $34.21 \pm 7.58$ & $36.44 \pm 4.73 \dagger$ & - & - & - \\
$L F n$ & $42.99 \pm 11.82$ & $38.42 \pm 8.22 \dagger$ & - & - & - \\
$H F n$ & $42.49 \pm 7.27$ & $44.16 \pm 4.84 \dagger$ & - & - & - \\
$L F / H F$ & $1.12 \pm 0.49$ & $0.92 \pm 0.2 \dagger^{\dagger}$ & - & - & - \\
\hline$A C[1,2]$ & $-6.72 \pm 3.29$ & $-3.80 \pm 1.65^{* *}$ & 83.3 & 56.7 & 0.811 \\
$D C[1,2]$ & $7.28 \pm 3.62$ & $3.95 \pm 1.64^{*}$ & 83.3 & 53.3 & 0.817 \\
$A C[3,10]$ & $-7.13 \pm 2.57$ & $-4.79 \pm 2.07^{*}$ & 66.7 & 63.3 & 0.758 \\
$D C[3,10]$ & $7.67 \pm 2.80$ & $5.10 \pm 2.37^{*}$ & 66.7 & 66.7 & 0.753 \\
\hline$A E[1]$ & $21.97 \pm 14.23$ & $13.71 \pm 3.34 \dagger$ & - & - & - \\
$D E[1]$ & $23.56 \pm 15.47$ & $13.99 \pm 3.44 \dagger$ & - & - & - \\
$A A[1]^{*} 10^{4}$ & $22.47 \pm 2.81$ & $19.59 \pm 1.84^{* *}$ & 83.3 & 56.7 & 0.833 \\
$D A[1]^{*} 10^{4}$ & $22.48 \pm 2.80$ & $19.59 \pm 1.85^{* *}$ & 83.3 & 60.0 & 0.836 \\
$A E[3]$ & $22.31 \pm 10.49$ & $14.39 \pm 4.96^{*}$ & 83.3 & 50.0 & 0.769 \\
$D E[3]$ & $23.93 \pm 11.56$ & $14.92 \pm 5.25^{*}$ & 75.0 & 50.0 & 0.783 \\
$A A[3]^{*} 10^{4}$ & $22.41 \pm 2.81$ & $19.54 \pm 1.83^{* *}$ & 83.3 & 60.0 & 0.842 \\
$D A[3]^{*} 10^{4}$ & $22.44 \pm 2.81$ & $19.56 \pm 1.84^{* *}$ & 83.3 & 60.0 & 0.833 \\
\hline \hline
\end{tabular}

meanRR, $\overline{S D N N, A C, D C, A E \text { and } D E \text { expressed in ms. } V L F n, L F n \text { and } H F n}$ expressed in n.u. $A A$ and $D A$ expressed in area units. ${ }^{* *}$-value $<0.005,{ }^{*}$-value $<0.05, \dagger p$-value $=$ n.s. 
687

688

689

690

691

692

693

694

695

696

697
TABLE VII

RR INTERVALS OF SLEEP PERIODS OF ECG RECORDINGS FROM LR AND HR PATIENTS

\begin{tabular}{llllll}
\hline \hline & $\begin{array}{l}\text { LR } \\
\text { mean } \pm \text { std }\end{array}$ & $\begin{array}{l}\text { HR } \\
\text { mean } \pm \text { std }\end{array}$ & $\begin{array}{l}\text { Sen } \\
(\%)\end{array}$ & $\begin{array}{l}\text { Spe } \\
(\%)\end{array}$ & AUC \\
\hline$N$ (subjs.) & 30 & 12 & & & \\
$m e a n R R$ & $957.0 \pm 136.2$ & $785.3 \pm 103.2 \ddagger$ & 66.7 & 80.0 & 0.867 \\
$S D N N$ & $93.2 \pm 42.5$ & $57.0 \pm 16.6 \$$ & 75.0 & 66.7 & 0.797 \\
\hline$V L F n$ & $33.29 \pm 7.65$ & $32.08 \pm 5.02 \dagger$ & - & - & - \\
$L F n$ & $42.85 \pm 9.52$ & $38.16 \pm 6.29^{*}$ & 83.3 & 60.0 & 0.650 \\
$H F n$ & $45.33 \pm 6.54$ & $45.68 \pm 5.05^{*}$ & 75.0 & 36.7 & 0.544 \\
$L F / H F$ & $1.03 \pm 0.36$ & $0.89 \pm 0.27 \dagger$ & - & - & - \\
\hline$A C[1,2]$ & $-10.79 \pm 4.73$ & $-5.92 \pm 2.27 \ddagger$ & 83.3 & 60.0 & 0.844 \\
$D C[1,2]$ & $11.44 \pm 5.25$ & $6.00 \pm 1.91 * *$ & 83.3 & 63.3 & 0.850 \\
$A C[3,10]$ & $-11.40 \pm 4.55$ & $-7.05 \pm 3.08^{* *}$ & 91.7 & 66.7 & 0.789 \\
$D C[3,10]$ & $12.06 \pm 4.96$ & $7.34 \pm 3.26^{* *}$ & 91.7 & 66.7 & 0.783 \\
\hline$A E[1]$ & $32.17 \pm 17.78$ & $19.63 \pm 6.77^{*}$ & 91.7 & 40.0 & 0.756 \\
$D E[1]$ & $34.06 \pm 18.79$ & $19.91 \pm 7.80^{*}$ & 83.3 & 43.3 & 0.772 \\
$A A[1]^{*} 10^{4}$ & $28.67 \pm 4.09$ & $23.51 \pm 3.08 \ddagger$ & 66.7 & 80.0 & 0.867 \\
$D A[1]^{*} 10^{4}$ & $28.64 \pm 4.06$ & $23.52 \pm 3.07 \ddagger$ & 66.7 & 80.0 & 0.867 \\
$A E[3]$ & $35.97 \pm 14.48$ & $22.58 \pm 7.98^{* *}$ & 91.7 & 56.7 & 0.783 \\
$D E[3]$ & $38.07 \pm 15.39$ & $23.42 \pm 8.48^{* *}$ & 91.7 & 56.7 & 0.799 \\
$A A[3]^{*} 10^{4}$ & $28.62 \pm 4.09$ & $23.49 \pm 3.08 \ddagger$ & 66.7 & 80.0 & 0.867 \\
$D A[3]^{*} 10^{4}$ & $28.63 \pm 4.08$ & $23.50 \pm 3.09 \ddagger$ & 66.7 & 80.0 & 0.867 \\
\hline \hline
\end{tabular}

meanRR, SDNN, $A C, D C, A E$ and $D E$ expressed in ms. $V L F n, L F n$ and $H F n$ expressed in n.u. $A A$ and $D A$ expressed in area units. $\$$ p-value $<0.0005, \S p$-value $<0.001,{ }^{*}$ p-value $<0.005,{ }^{*}$-value $<0.05$, $\dagger$ pvalue $=$ n.s. 